Supporting Information

\title{
Silver Nanowire-Bacterial Cellulose Composite Fiber-Based Sensor for Highly Sensitive Detection of Pressure and Proximity
}

Fangyi Guan,,${ }^{1,2 \dagger}$ Yu Xie, ${ }^{1 \dagger}$ Hanxiang Wu, ${ }^{1}$ Yuan Meng,,${ }^{1}$ Ye Shi, ${ }^{1}$ Meng Gao, ${ }^{1}$ Ziyang Zhang, ${ }^{1}$ Shiyan Chen, ${ }^{2}$ Ye Chen, ${ }^{2}$ Huaping Wang, ${ }^{2}$ and Qibing Pei $^{1 *}$

${ }^{1}$ Department of Materials Science and Engineering, Henry Samueli School of Engineering and Applied Science, University of California, Los Angeles, CA 90095, USA

${ }^{2}$ State Key Laboratory for Modification of Chemical Fibers and Polymer Materials, College of Materials Science and Engineering, Donghua University, Shanghai 201620, PR China

$\dagger$ These authors contributed equally to the work

*Corresponding author. Email: qpei@ seas.ucla.edu 

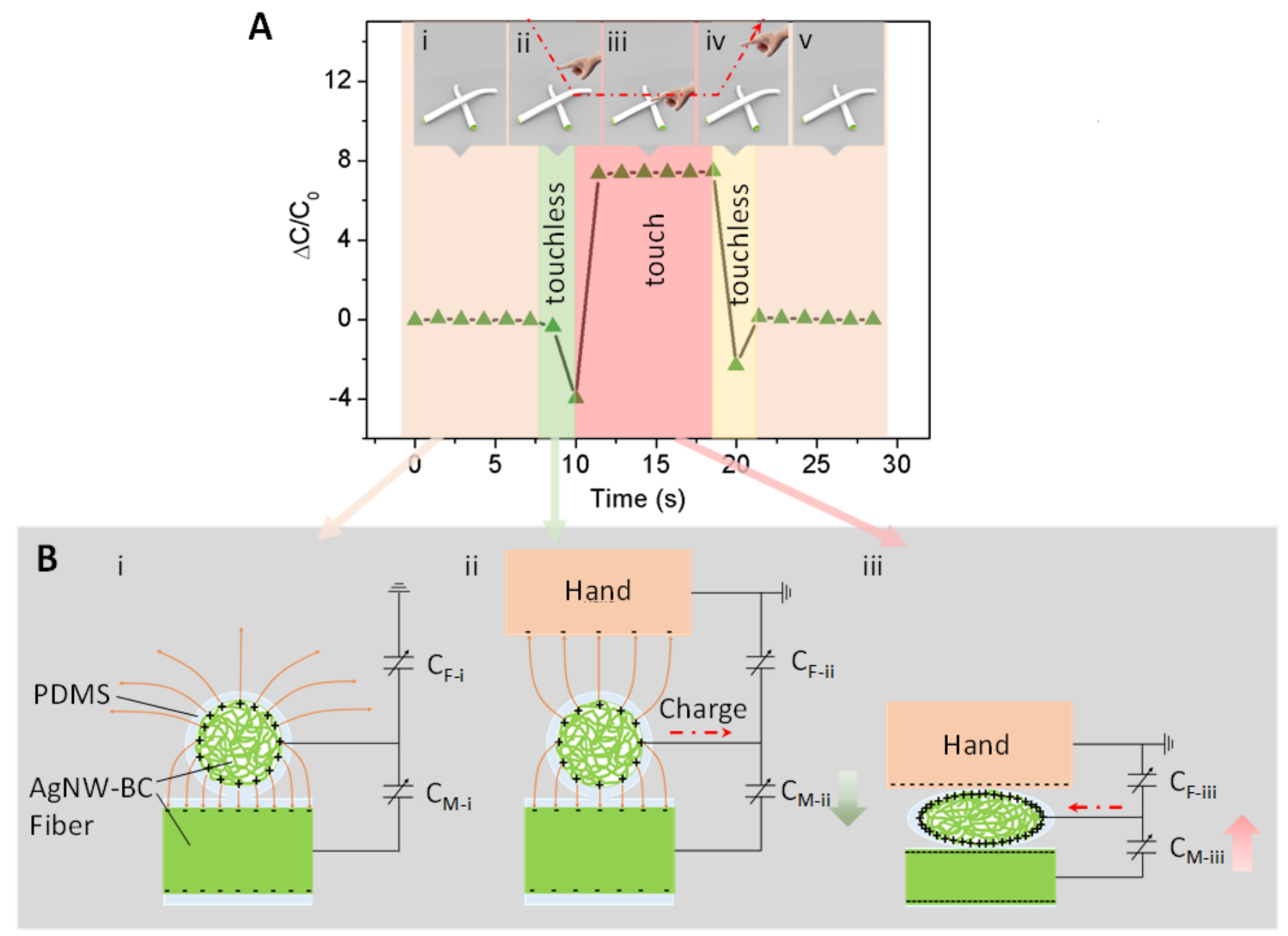

Figure S1. Bimodal sensing of the AgNW-BC/PDMS fiber sensor. (A) Capacitive response to removable hand when it is approaching (ii, light green area), touching (iii, light red area) and leaving (iv, light yellow area) the sensor. The state before (i) and after (v) the presence of the hand are shown in the light orange area. (B) Distribution of the electronic field and charges (sectional view) on the fiber electrodes and equal circuit when the hand is out of proximity sensing range (i), approaching (ii) and pressing on the sensor (iii).

Our bimodal sensor was designed upon a fiber capacitor, built with two crossed porous AgNW-BC/PDMS fibers (Figure S1a-i). The overlap is a sensing point, considered as a parallel-plate capacitor. As illustrated in Figure S1, the fiber sensor could track a conductor at its approach, touch and departure (Figure S1a, red dashed arrow) by measuring the variation of capacitance. Here a human hand was taken as the example to be a conductor or operator. When a hand gradually approached, the fiber sensor responded rapidly with relative capacitance decreasing dramatically (Figure S1a-ii, light green area). Once it is close enough to 
touch the sensor, a sharp increase of capacitance was induced (Figure S1a-iii, light red area). Moving away the hand, the value of capacitive signals experienced a temporary decrease (Figure S1a-iv, light yellow area) and subsequent recovery to baseline (Figure S1a-v, light orange area). The bimodal sensing mechanism is based on two types of capacitance induced by a parallel-plate capacitor: mutual capacitance $\left(\mathrm{C}_{\mathrm{M}}\right)$ between the two electrodes and self-capacitance to the ground (fringe capacitance, $\mathrm{C}_{\mathrm{F}}$ ) (Figure $\mathrm{S} 1 \mathrm{~b}$ i). ${ }^{1-3}$ The dual function of the device was activated by changes in $\mathrm{C}_{\mathrm{M}}$ or flow of electrical charges.

In touchless sensing (Figure S1a-ii), approaching hand (or conductive/charged objects) acted as a third electrode, partially intercepting and shunting the fringing electric field to the ground within a detectable range (Figure S1b-ii). The hand capacitively coupled to the sensor element, resulting in a decrease of coupling between the two electrodes. ${ }^{1}$ For the increased electric field directed toward the approaching objects, charges on the two electrodes flew out resulting in an increase of $\mathrm{C}_{\mathrm{F}}$ and the electrodes are discharged. ${ }^{4}$ It caused a logarithmical drop of $\mathrm{C}_{\mathrm{M}}$, corresponding well with the model developed by Garbini: ${ }^{5}$

$$
C=\left(4 \varepsilon^{\prime} W \ln (2 H / h)\right) / \pi
$$

where $W$ and $H$ refer to the geometry width and height of the approaching conductor, $h$ is the distance between the bottom of the conductor and the top electrode, and $\varepsilon^{\prime}$ is a constant factor. As a key index of senor performance, the sensitivity of the proximity sensor $\left(\mathrm{S}_{\mathrm{pro}}\right)$ is defined as:

$$
S_{\text {pro }}=\delta\left(\Delta C / C_{0}\right) / \delta h=\left(\operatorname{lnh} h_{0}-\ln h\right) /\left(\ln 2 H-\ln h_{0}\right)
$$

Where $\mathrm{C}_{0}$ and $\mathrm{h}_{0}$ are the initial capacitance and distance between the bottom of the conductor or hand and the top electrode. No hint is provided for the better design of the sensor, because the equation shows no linking between factors of device and proximity sensitivity. But a smaller overlap sensing area has been demonstrated to be capable of providing improved projection of electric field and enhanced proximity sensing performances. ${ }^{1}$ It provides insights into the optimal design of the highly sensitive proximity sensor - building fiber electrodes with thin diameter. This is consistent with the requirement of unobtrusiveness for the wearable device.

On the other hand, pressure sensing of the device is induced by mechanical force, resulting in increase of $\mathrm{C}_{\mathrm{M}}$ (Figure S1a-iii). It can be easily explained with the following equation: 


$$
C=\varepsilon A / 4 \pi k d
$$

where $\mathcal{E}$ is dielectric constant, $\mathrm{A}$ is the effective overlap area, $\mathrm{k}$ is electrostatic constant, and $\mathrm{d}$ is the distance between the two AgNW-BC fiber electrodes. When a tactile pressure is applied to the overlap of the two fibers, the compression of electrodes or/and dielectric layer leads to an increase of $A$ and $E$ and decline of $d$, inducing the positive variations of the capacitance of the sensor. Sensitivity ( $S_{\text {pre }}$ of a capacitive pressure sensor is generally defined as:

$$
S_{\text {pre }}=\delta\left(\Delta C / C_{0}\right) / \delta P=A / d A_{0}-1 / d_{0}
$$

where $\mathrm{P}$ indicates the applied pressure $(\mathrm{Pa})$, and $\mathrm{C}(\mathrm{F})$ represents the capacitances with applied pressure, respectively. $\mathrm{C}_{0}, \mathrm{~A}_{0}$ and $\mathrm{d}_{0}$ are the initial capacitance, effective overlap area and the distance between electrodes when $\mathrm{P}=0$. High sensitivity requires the electrodes or dielectric layers with sharp deformation even under subtle external force. ${ }^{6}$ Constructing microstructure electrodes and dielectric layers is considered the most effective method. ${ }^{6}$ A highly porous dielectric layer has been introduced to achieve high sensitive capacitive fabrics sensor. ${ }^{6}$ However, the method is unrealistic for thin fiber aiming at the unobtrusive sensor, because the ultrathin dielectric layers may lead to complex fabrication. Microporous fiber electrodes is a potential approach to realize high sensitivity of thin fiber sensor since external pressure induces not only decrease of $\mathrm{d}$ for thinner dielectric layers, but the deformation of the porous fiber with air being extruded, increasing the effective area A proportionally (Figure S1b-iii). With both the decrease of $\mathrm{d}$ and increase of $\mathrm{A}$, high sensitivity is promising to be achieved according to the equation derivated from equation (S4).

Therefore, a highly porous but thin fiber electrode is a promising candidate for a highly sensitive bimodal capacitive fiber sensor with a high area variation for capacitance change and improved projection of the electric field. For the fabrication of the fiber electrodes, the properties and geometry of the fibers largely depends on the wet-spinning process. Excessive BC nanofibers would obstruct the electrically conductive path of AgNW, resulting in inferior conductivity. Inversely, superabundant AgNW could cause defective structure, poor mechanical performance and even failure to maintain a fibrous structure. Higher stretching ratio leads to smaller fiber diameter; whereas diluted spinning suspension generates increased number and size of pores of the fibers. 

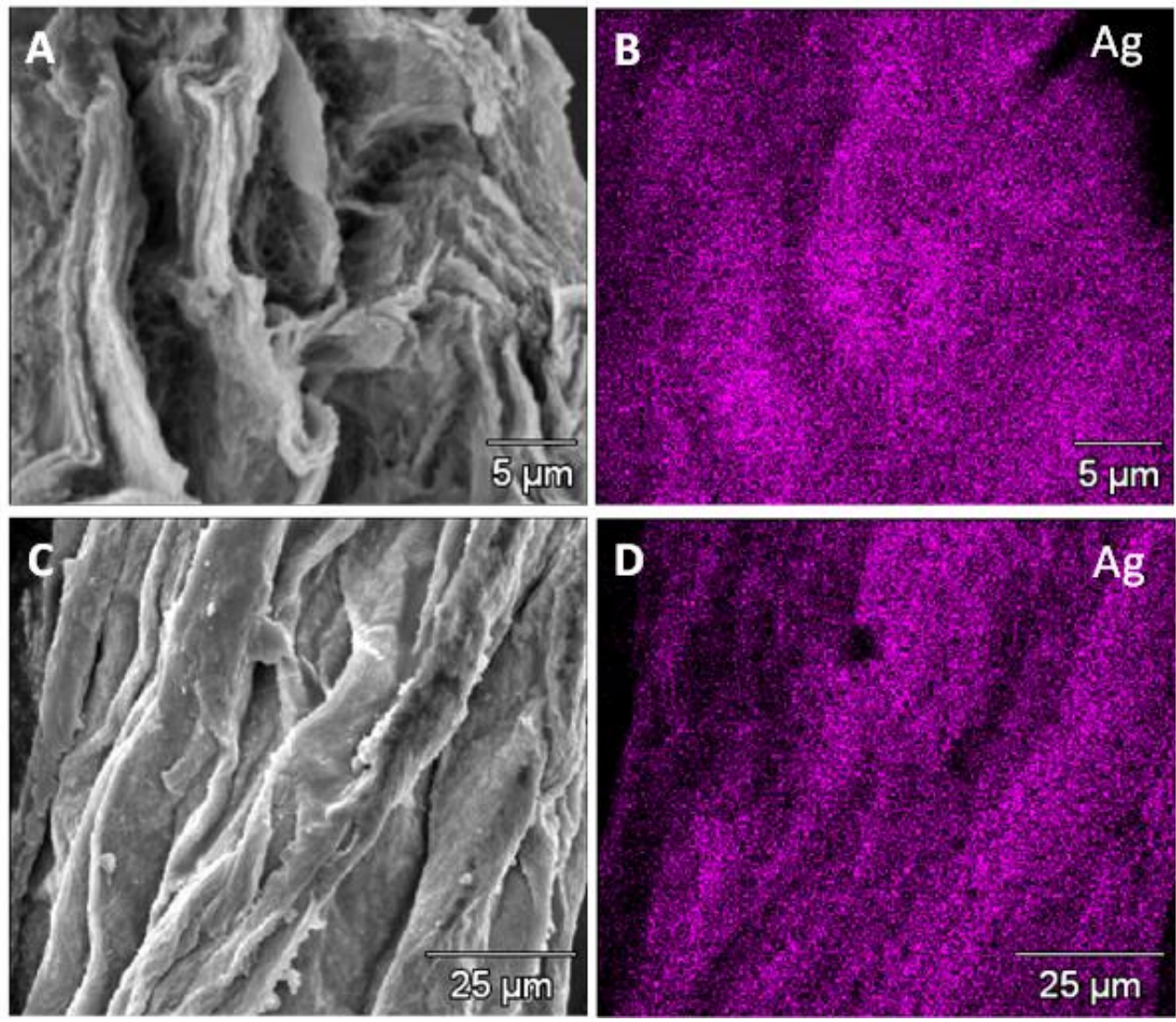

Figure S2. Dispersion of AgNW in AgNW-BC fibers. Cross-sectional (A) and surface (C) SEM images and the corresponding EDS mapping images (B and $\mathbf{D})$ of the AgNW-BC fiber. The cross-sectional EDS mapping shows the spatial distribution of $\mathrm{Ag}$ in the conductive fiber, revealing that the $\mathrm{Ag}$ were well distributed inside as well as on the surface of the AgNW-BC conductive fiber along with its hierarchical structure. 


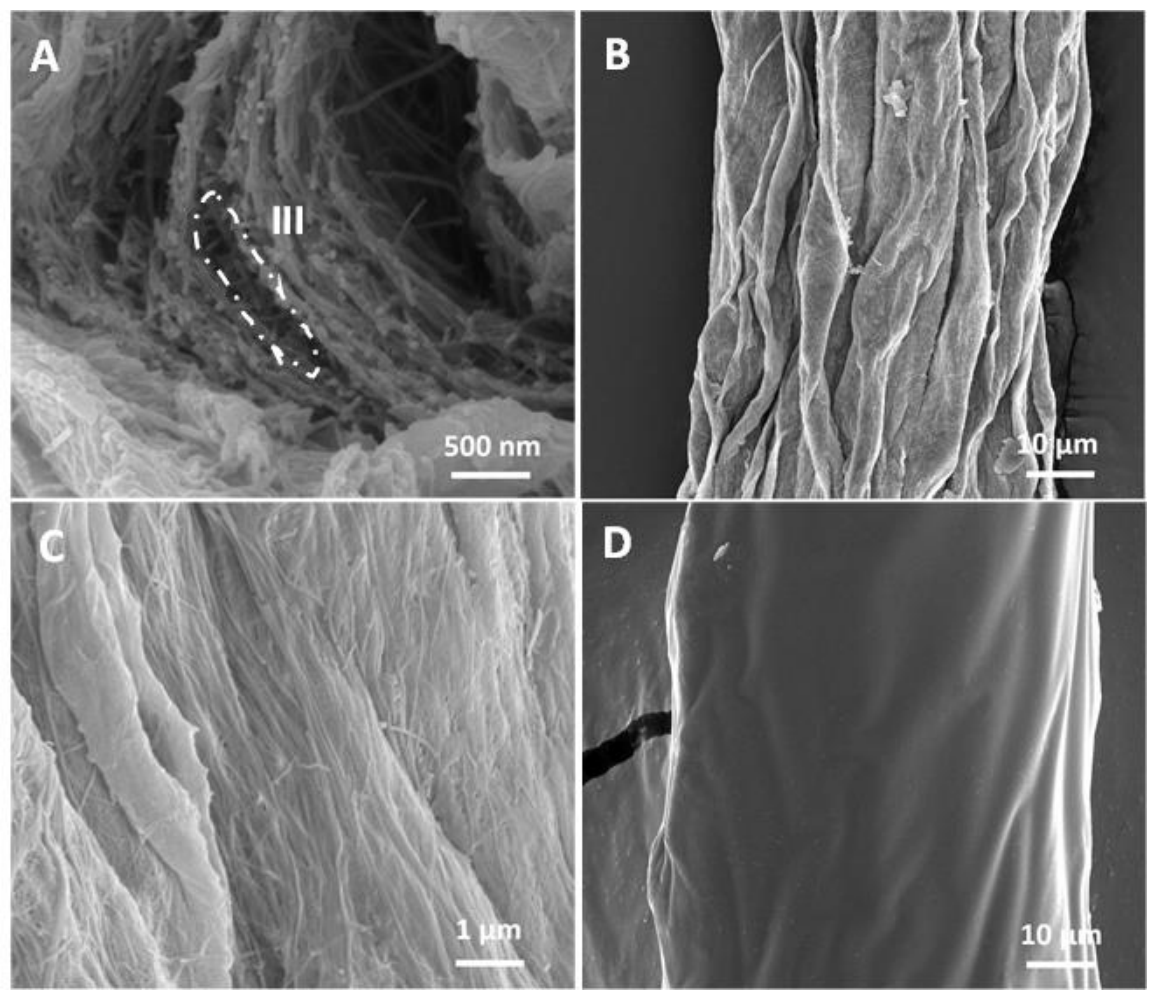

Figure S3. Microstructure of AgNW-BC and AgNW-BC/PDMS. Cross-section (A) and surface (B, C) SEM images of AgNW-BC fibers. (D) Surface SEM of an AgNW-BC/PDMS fiber.

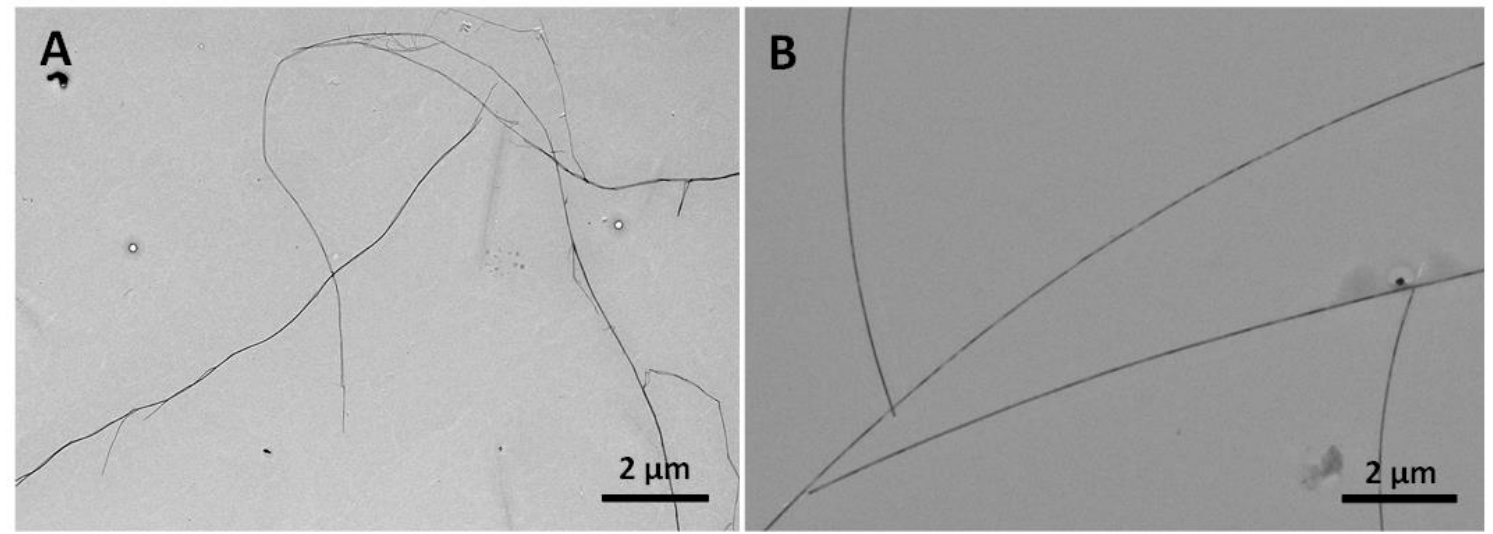

Figure S4. Morphology of raw components. Transmission electron microscope (TEM) of BC nanofibers (A) and AgNWs (B). 


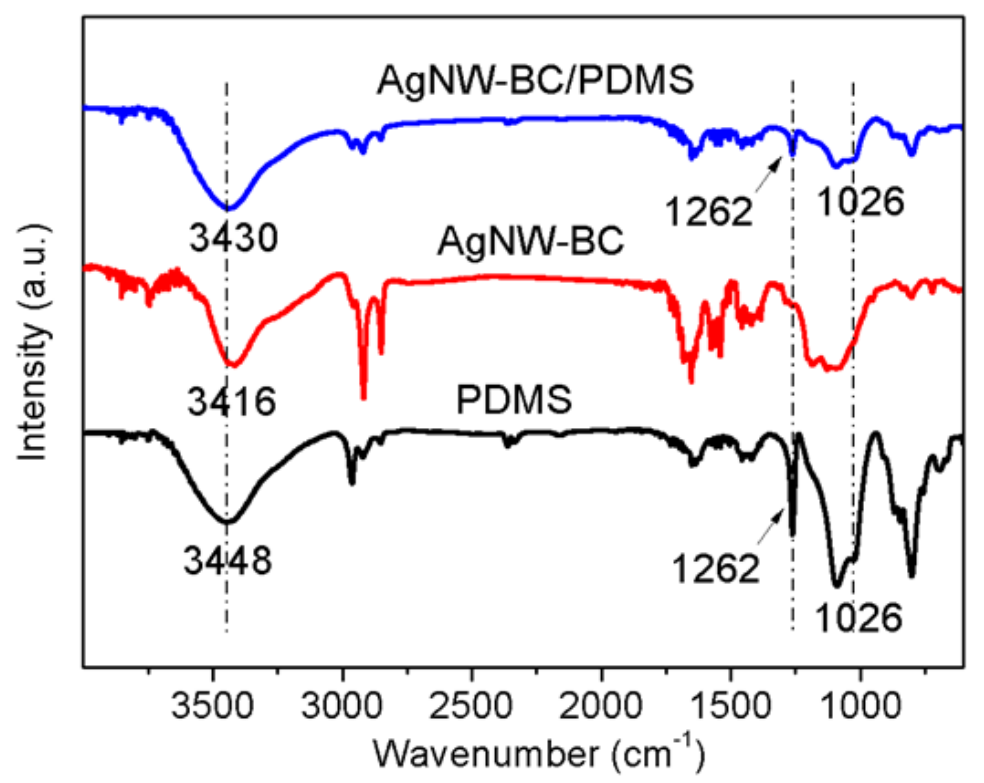

Figure S5. Interaction between AgNW-BC fibers and PDMS layer in AgNW-BC/PDMS fiber. Fourier Transform Infrared Spectroscope (FTIR) of PDMS film, AgNW-BC fiber and AgNW-BC/PDMS fiber.

New absorbing peaks appeared in the FTIR spectroscopy of the obtained AgNW-BC/PDMS fiber compared with that of the AgNW-BC fiber (Figure S5), including featured peaks of the $\mathrm{Si}_{-} \mathrm{CH}_{3}$ stretching vibration $\left(1262 \mathrm{~cm}^{-1}\right)$ and $\mathrm{Si}-\mathrm{O}$ stretching vibration $\left(1026 \mathrm{~cm}^{-1}\right) .{ }^{7}$ Besides, the $\mathrm{O}-\mathrm{H}$ stretching vibration peaks shifted from $3416 \mathrm{~cm}^{-1}$ to $3430 \mathrm{~cm}^{-1}$, which may be ascribed to the interaction between AgNW-BC fiber and PDMS or the overlay of the 3416 and 3448 peaks. It demonstrated that PDMS has been attached successfully to the core AgNW-BC. 
A

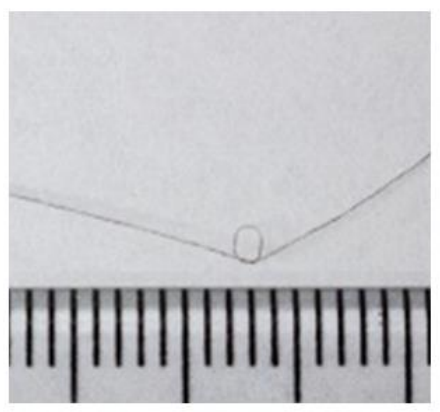

B

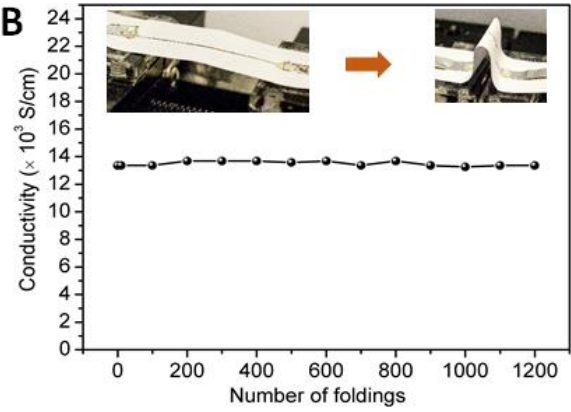

C

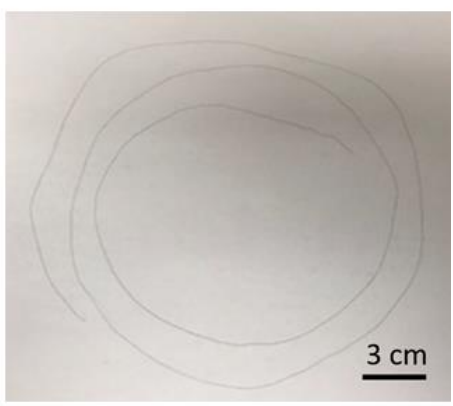

Figure S6. Properties of AgNW-BC fiber. (A) An AgNW-BC fiber knotted into a circle with a curvature radius of $1.5 \mathrm{~mm}$. (B) Endurance of conductivity within 1200 bending cycles with a curvature radius of $1.5 \mathrm{~mm}$. (C) Continuous long AgNW-BC fiber with a length of $40 \mathrm{~cm}$.

Flexibility and toughness are significant properties for wearability of e-skins. AgNW-BC fibers were capable to be knotted into a circle with a radius of less than $1 \mathrm{~mm}$, demonstrating the flexibility of the fibers. Since stability and constant conductivity of the fibers are essential for wearable devices in longterm practical usage, a bending-unbending experiment was conducted to identify the conductive durability (Figure S6B). After 1200 cycles of bending to a curvature radius of $1.5 \mathrm{~mm}$, the AgNW-BC fiber maintained its initial conductivity. To meet the requirement of scalability of industry, AgNW-BC fibers with a length of more than $40 \mathrm{~cm}$ was spun continuously (Figure S6C), indicating the potential in scaleup production. 

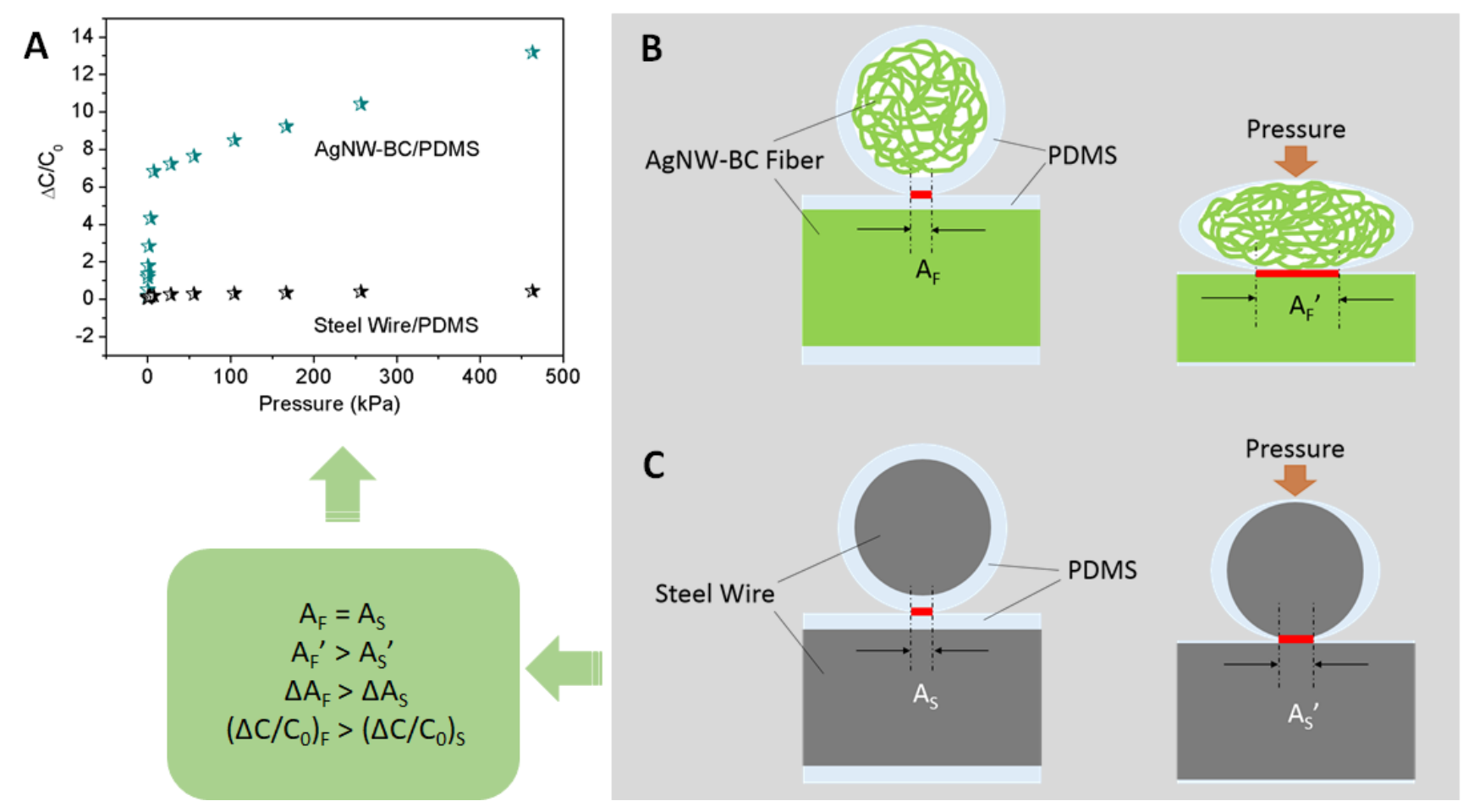

Figure S7. Mechanism of porous structure performing good sensitivity. (A) Relative capacitance changes of steel wire/PDMS and AgNW-BC/PDMS sensor. Mechanism of capacitance changes of AgNW-BC/PDMS (B) and steel wire/PDMS (C) fiber-based sensor.

The sensitivity of the AgNW-BC/PDMS fiber sensor is superior to that of steel wire/PDMS (Figure S7A) due to the different ability of the formation of the conductive cores. As described in the scheme of Figure S7B, applied with pressure, the overlap area As of steel wire/PDMS sensor stayed almost the same for the incompressible solid steel wire and thus $\Delta \mathrm{A}_{\mathrm{S}}$ is approximate to 0 . In comparison, the change of $\mathrm{A}_{\mathrm{F}}$ is rather obvious, $\Delta \mathrm{A}_{\mathrm{F}}$ being far more than $\Delta \mathrm{A}_{\mathrm{s}}$ and resulting in better sensing performance. 

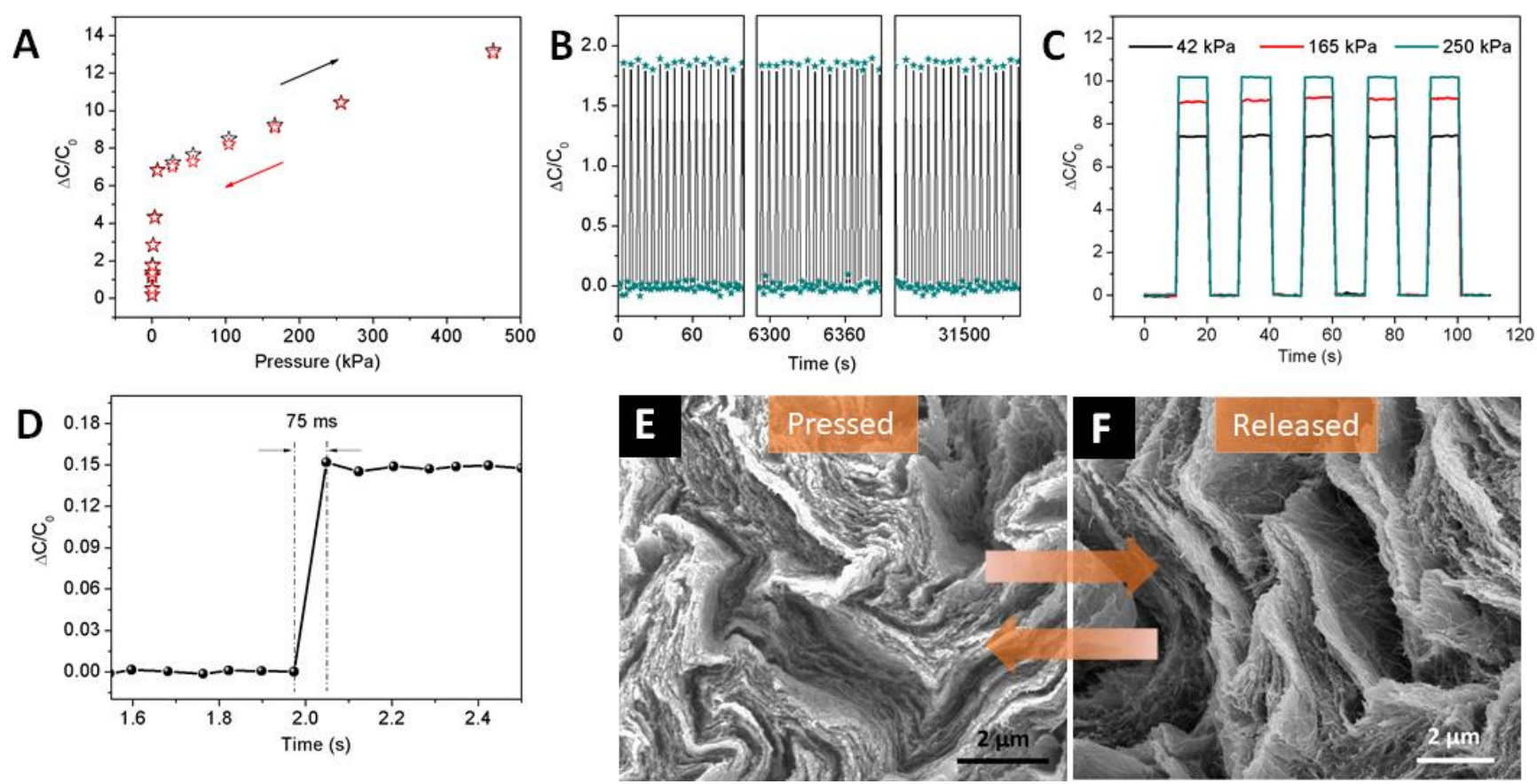

Figure S8. Pressure sensing performance and the corresponding change in the microstructure of the AgNW-BC/PDMS fibers. (A) Relative capacitance change of the fiber sensor with the loadingunloading cycles. (B) Durability characterization for the fiber sensor under a loading pressure of $600 \mathrm{~Pa}$ over about 2000 cycles. (C) Capacitance analysis of time by periodic loads of 42, 165 and $250 \mathrm{kPa}$ over multiple test cycles. (D) Response time of the AgNW-BC/PDMS sensor to pressure. (E,F) Core structure of an AgNW-BC/PDMS fiber being compressed radially and after releasing, repeatedly. 

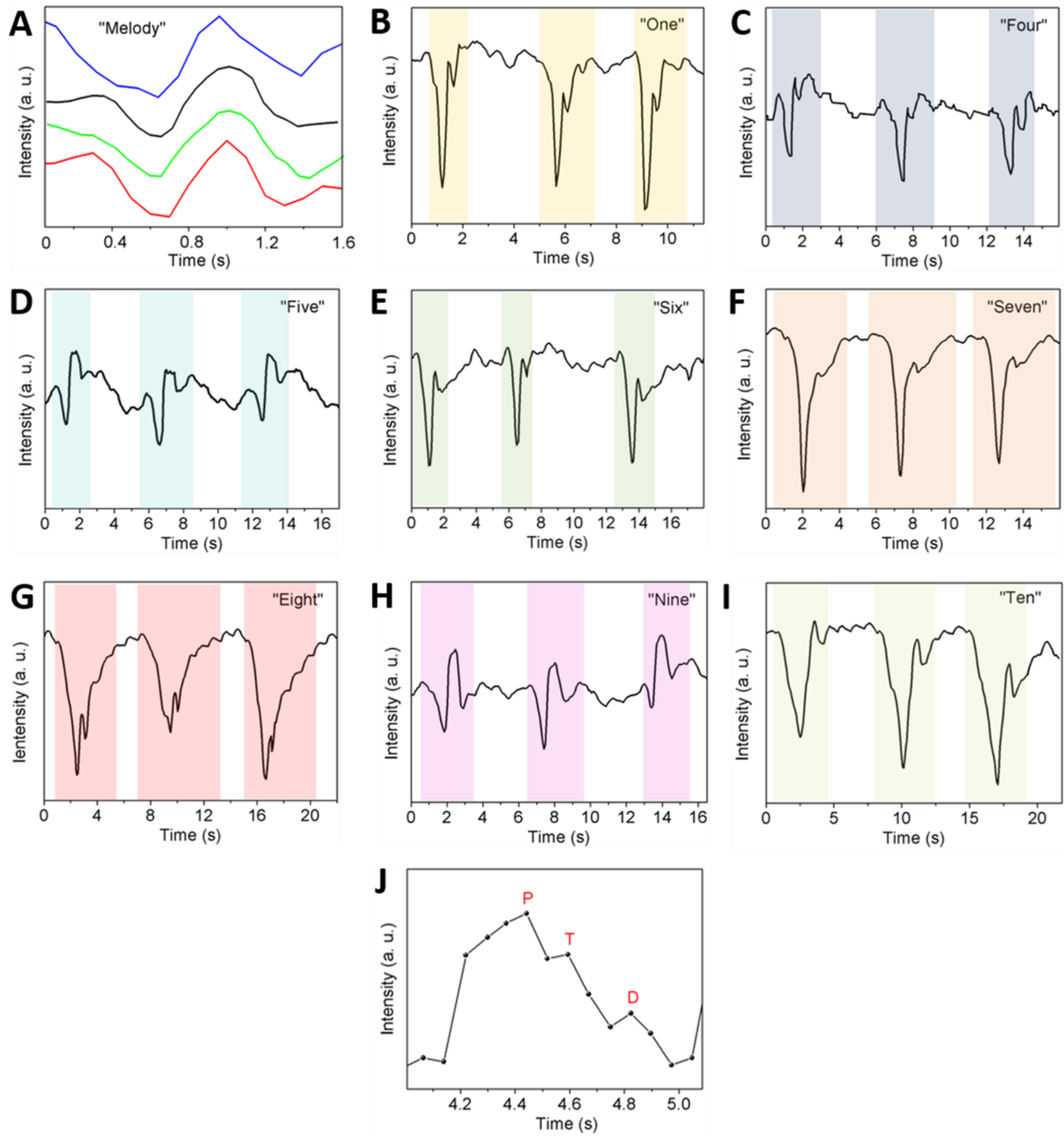

Figure S9. Voice and pulse recognition. Capacitance curves of AgNW-BC/PDMS fiber sensor in response to the name "Melody" (A) and pronunciations of numbers $(\mathbf{B}-\mathbf{I})$. (J) Relative capacitive change sensing one pulse of a female volunteer.

\section{Machine Learning Classification Model for Phonation Recognition}


Data collection. The pressure sensor was mounted on the throat of a female test subject. The voice waveforms of number 1-10 were collected through the pressure sensor by letting the same test subject speaking each number 10 times. Capacitance v.s. time data was recorded for each number at the frequency of $10 \mathrm{~Hz}$, and the capacitance data were labelled manually.

Data Pre-processing. The raw voice waveforms were first selected manually to filter out abnormal data. The resulted dataset has 93 valid inputs. Then the whole dataset was formatted based on the tsfresh formatting rule. Tsfresh is a python package to automatically calculate time series characteristics. 285 relevant features of the 1-10 digit voice waveforms were extracted through tsfresh augmenter. The feature data frame was then normalized with zero mean and unit variance. Now the normalized feature dataset was ready to be fed into classifiers.

Baseline. Before feeding the feature dataset into classifiers, a baseline classification was performed without feature extraction. Due to each digit voice waveform input has different total time length, another time series python package, tslearn, was used to transform all the input data into the same shape. The transformed dataset has shape of $(93,40)$. The transformed dataset was also normalized with zero mean and unit variance before use. Random forest classifier was selected due to its good performance. After a grid search with 6-fold cross-validation, the best result achieved with 200 trees and 45 maximum depth for each tree. The grid search results are shown Figure S10. And the best prediction accuracy result for this classifier is 0.7154 and the corresponding mean squared error is 4.0318 .
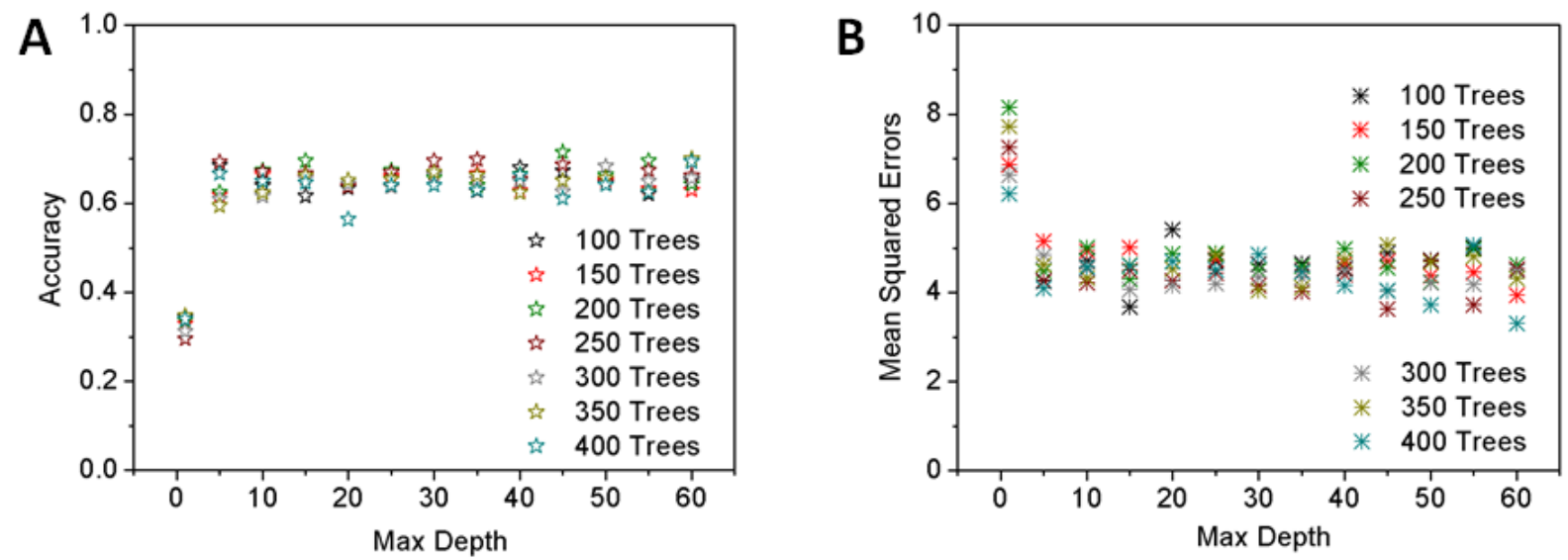

Figure S10. Grid search results of baseline random forest model without feature extractions. The accuracy (A) and mean squared errors (B) with different numbers (100, 150, 200,250, 300, 350 and 400) of trees at various maximum depth $(1,5,10,15,20,25,30,35$ and 40). 
Classification. The same random forest classifier was selected for the pre-processed data with engineered features. After a grid search with 6-fold cross-validation, the best result achieved with 150 trees and 35 maximum depth for each tree. The grid search results are shown Figure S11. The best prediction accuracy result on the feature dataset is 0.9073 , which is $26.8 \%$ better than the prediction results for the dataset without feature selection. This high accuracy result also shows the potential for our pressure sensor to be used in voice detection application, since it can recognize and distinguish small pressures that come from different voice waveforms.
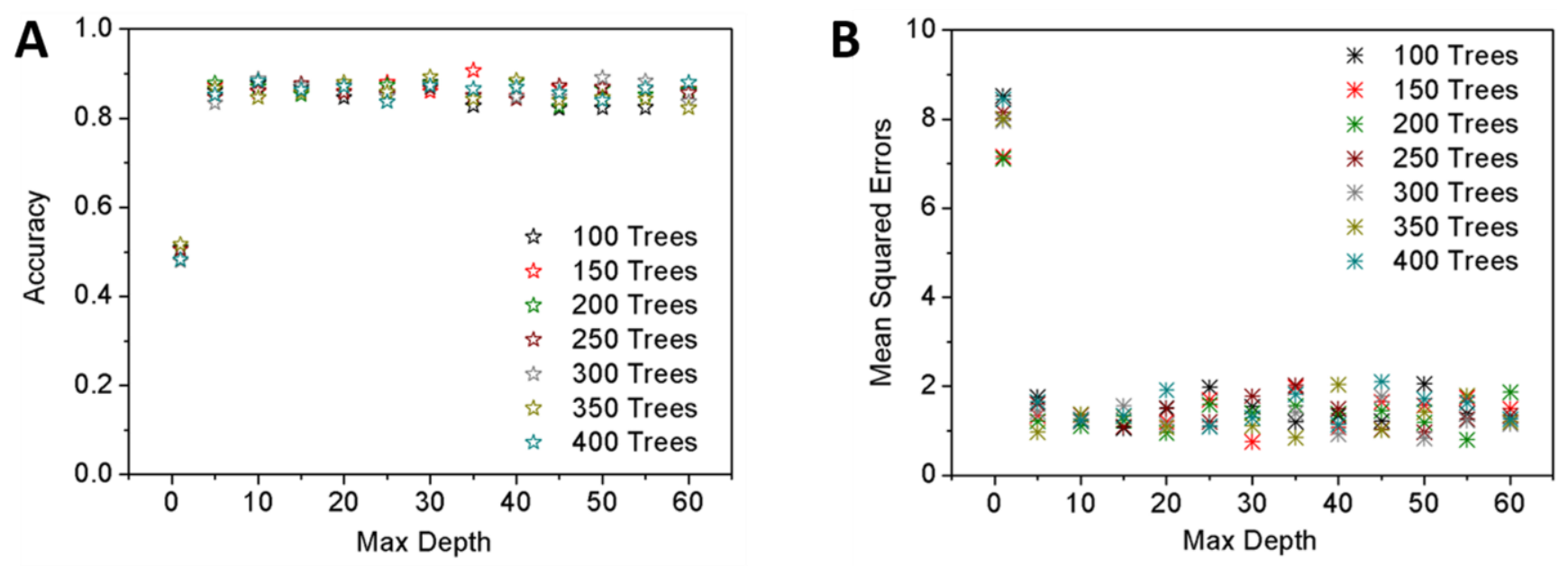

Figure S11. Grid search results of random forest model with feature extractions. The accuracy (A) and mean squared errors $(\mathbf{B})$ with different numbers $(100,150,200,250,300,350$ and 400) of trees at various maximum depth $(1,5,10,15,20,25,30,35$ and 40$)$. 


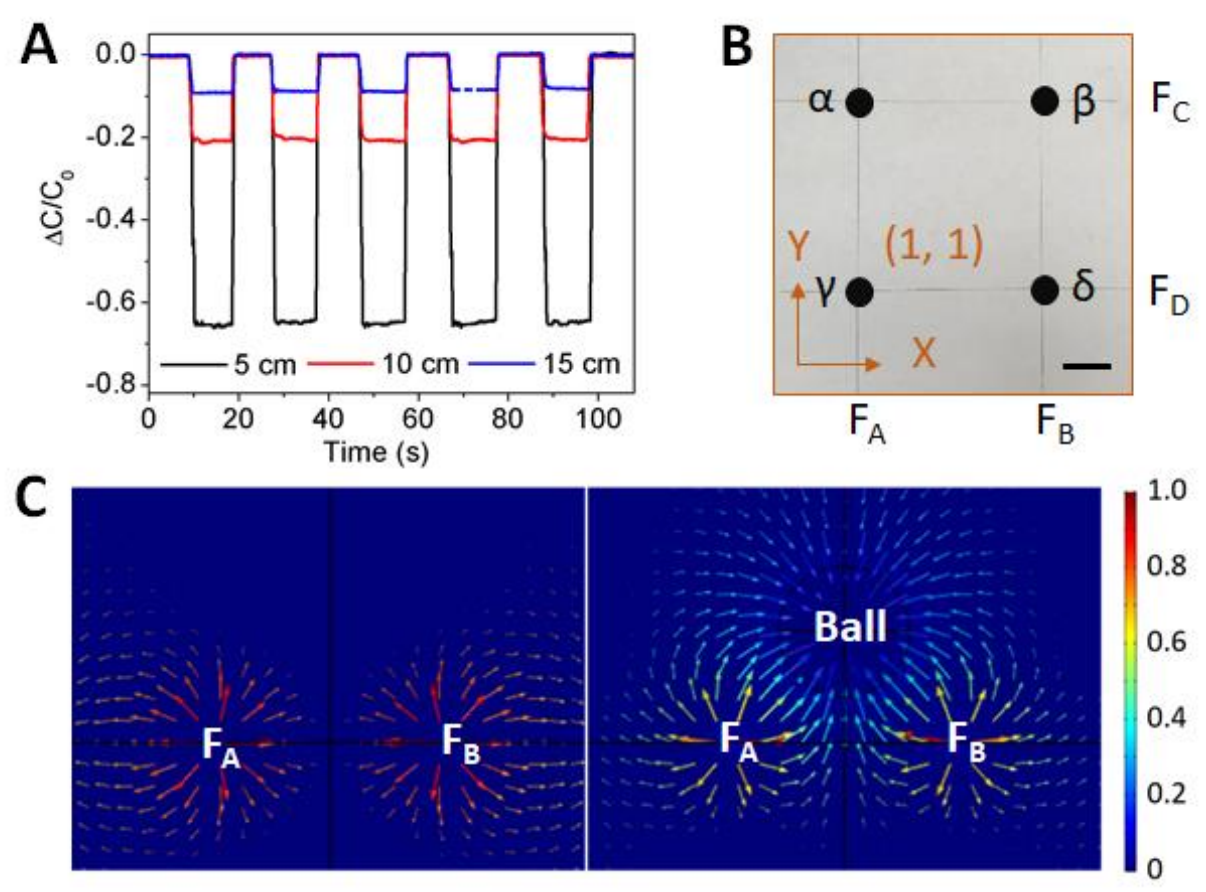

Figure S12. Proximity performance and mechanism. (A) The capacitive changes of the proximity sensor in response to a human hand placed 5, 10 and $15 \mathrm{~cm}$ above, in five consecutive cycles. (B) One crossover area of the multichannel panel $(2 \times 2)$ for calibration. The scale bar is $1 \mathrm{~cm}$. The four fibers cross over each other forming four intersections $(\alpha, \beta, \gamma$, and $\delta)$. Point $\gamma$ is defined as $(1,1)$ and the right direction is set as $\mathrm{X}$-axis and upward direction as $\mathrm{Y}$-axis. (C) COMSOL simulated electrical field distribution of two parallel fibers A and B connected to the positive pole of the power source without (i) and with (ii) the presence of a stainless ball at the point $(4 \mathrm{~cm}, 4 \mathrm{~cm}, 4 \mathrm{~cm})$. The rainbow-colored bar represents the relative magnitude of the electronic field at certain points. 

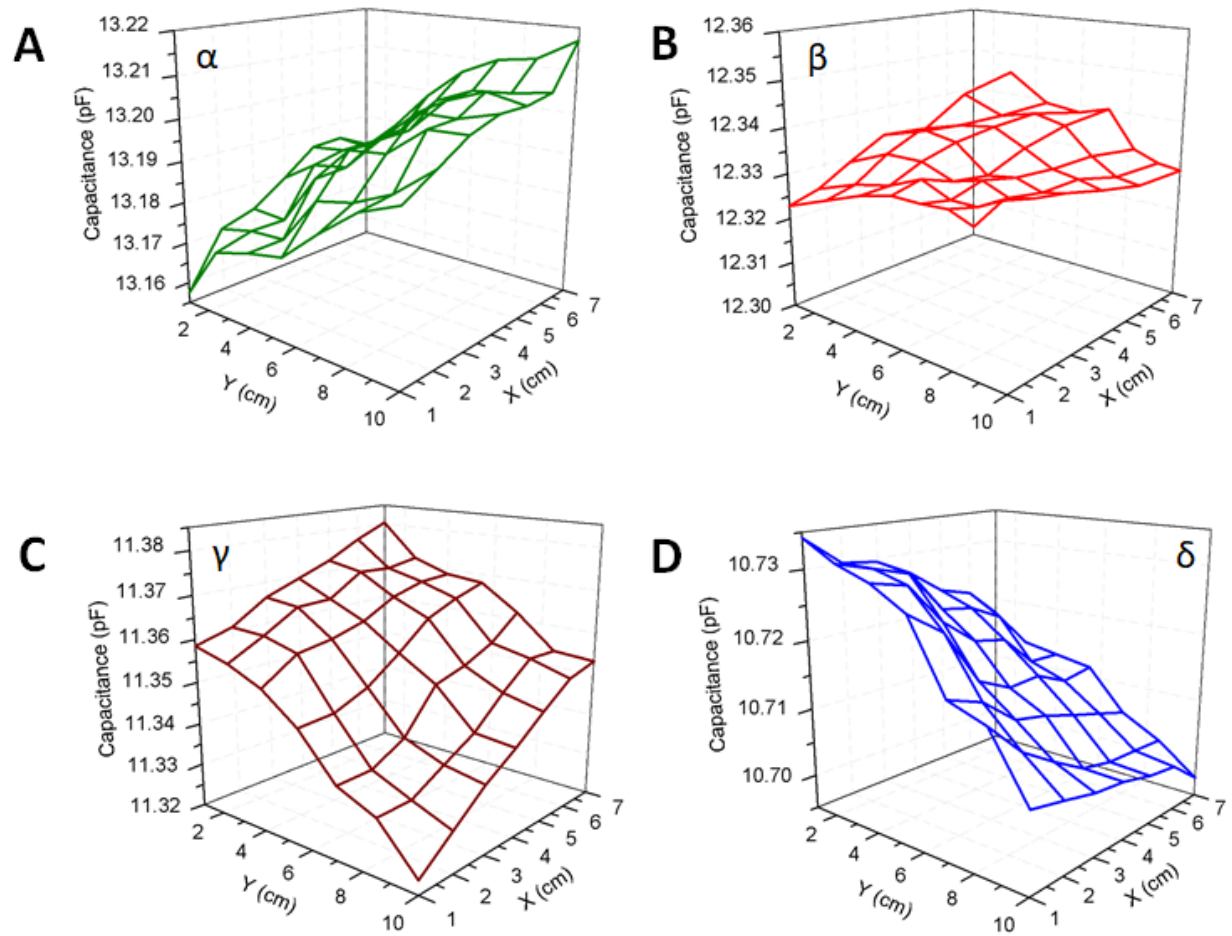

Figure S13. Rotational symmetric standard curves calibrated at a height of $4 \mathbf{c m}$. The rotational symmetric calibration grids $\mathrm{A}, \mathrm{B}, \mathrm{C}$ and $\mathrm{D}$ of the $2 \times 2$ sensor, corresponding to its four sensing point $\alpha, \beta$, $\gamma$ and $\delta$, respectively, with the metal ball at $4 \mathrm{~cm}$ above the senor.

A

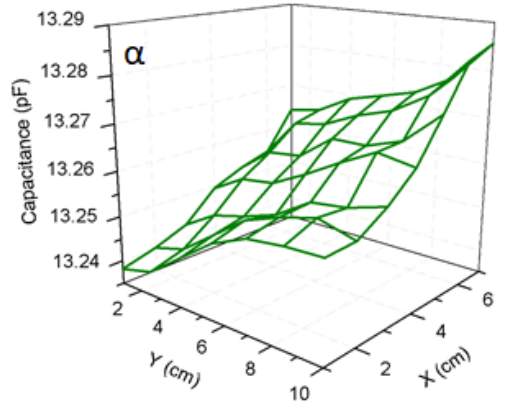

C

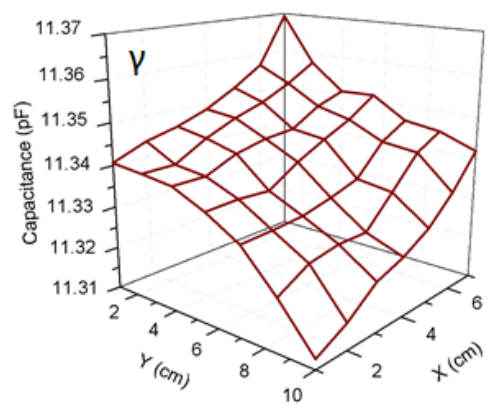

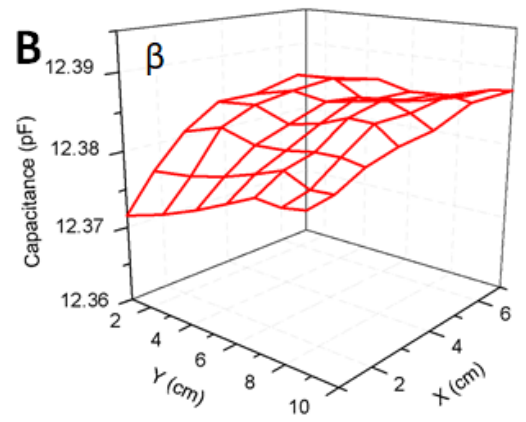

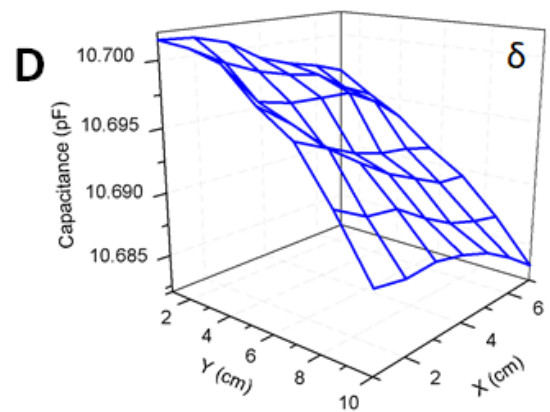


Figure S14. Rotational symmetric standard curves calibrated at a height of $5 \mathbf{c m}$. The rotational symmetric calibration grids $\mathrm{A}, \mathrm{B}, \mathrm{C}$ and $\mathrm{D}$ of the $2 \times 2$ sensor, corresponding to its four sensing point $\alpha, \beta$, $\gamma$ and $\delta$, respectively, with the metal ball at $5 \mathrm{~cm}$ above the senor.
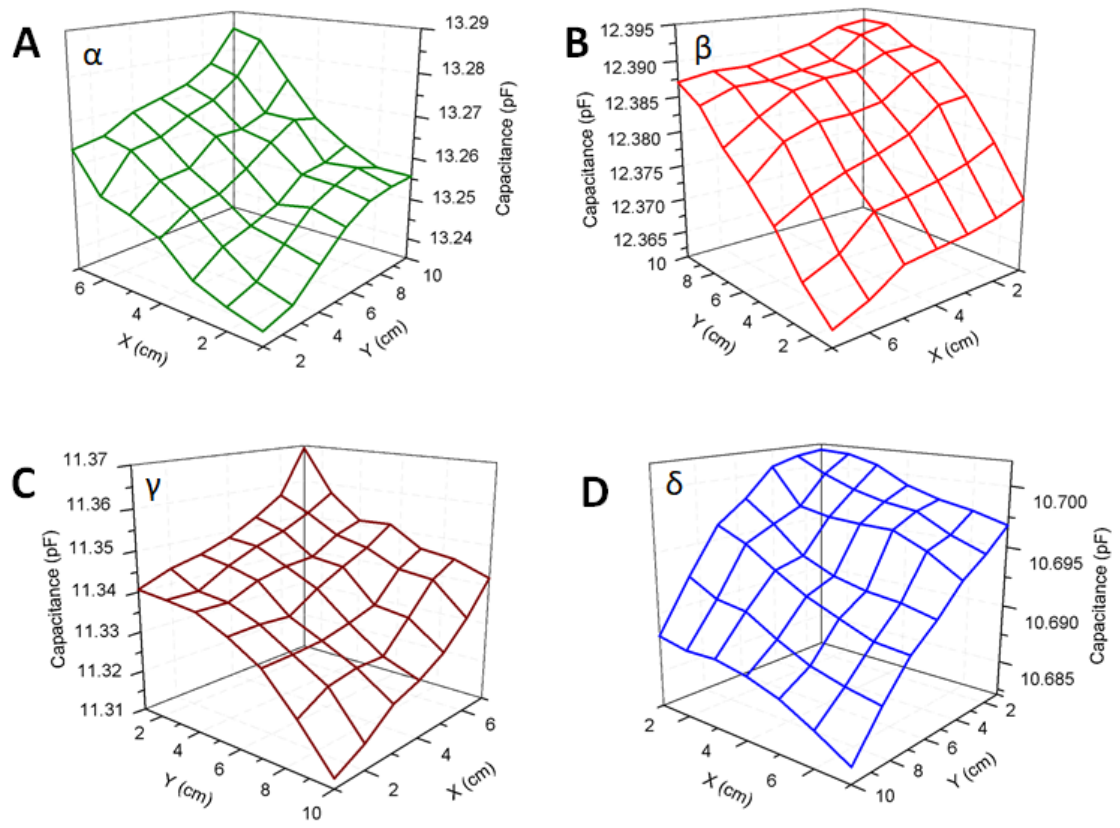

Figure S15. Frontal rotational symmetric standard curves calibrated at a height $0 \mathbf{5 m}$. The frontal rotational symmetric calibration grids A, B, C and D of the $2 \times 2$ sensor, corresponding to its four sensing point $\alpha, \beta, \gamma$ and $\delta$, respectively, with the metal ball at $5 \mathrm{~cm}$ above the senor.

\section{REFERENCES}

(1) Zhang, C.; Liu, S.; Huang, X.; Guo, W.; Li, Y.; Wu, H. A Stretchable Dual-Mode Sensor Array for Multifunctional Robotic Electronic Skin. Nano Energy 2019, 62, 164-170.

(2) Wang, J.; Lu, T.; Yang, M.; Sun, D.; Xia, Y.; Wang, T. Hydrogel 3D Printing with the Capacitor Edge Effect. Sci. Adv. 2019, 5, eaau8769.

(3) Lee, H.-K.; Chang, S.-I.; Yoon, E. Dual-Mode Capacitive Proximity Sensor for Robot Application: Implementation of Tactile and Proximity Sensing Capability on a Single Polymer Platform Using Shared Electrodes. IEEE Sens. J. 2009, 9, 1748-1755. 
(4) Sarwar, M. S.; Dobashi, Y.; Preston, C.; Wyss, J. K. M.; Mirabbasi, S.; Madden, J. D. W. Bend, Stretch, and Touch: Locating a Finger on an Actively Deformed Transparent Sensor Array. Sci. Adv. 2017, 3, e1602200.

(5) Garbini, J. L.; Saunders, R. A.; Jorgensen, J. E. In-Process Drilled Hole Inspection for Aerospace Applications Precis. Eng. 1991, 13, 125-134.

(6) Shuai, X.; Zhu, P.; Zeng, W.; Hu, Y.; Liang, X.; Zhang, Y.; Sun, R.; Wong, C.-P. Highly Sensitive Flexible Pressure Sensor Based on Silver Nanowires-Embedded Polydimethylsiloxane Electrode with Microarray Structure. ACS Appl. Mater. Interfaces 2017, 9, 26314-26324.

(7) Atayde, C. d. M.; Doi, I. Highly Stable Hydrophilic Surfaces of PDMS Thin Layer Obtained by UV Radiation and Oxygen Plasma Treatments. Phys. Status Solidi C 2010, 7, 189-192. 